\title{
Age-related differences in gastrocnemii muscles and Achilles tendon mechanical properties in vivo
}

\author{
India Lindemann ${ }^{1}$, Brooke K. Coombes ${ }^{2}$, Kylie Tucker ${ }^{1}$, Francois Hug ${ }^{1,3,4}$, Taylor J.M. Dick ${ }^{1 *}$ \\ ${ }^{1}$ School of Biomedical Sciences, The University of Queensland, Brisbane, Queensland, Australia \\ ${ }^{2}$ School of Allied Health Sciences -Griffith University, Brisbane, Australia \\ ${ }^{3}$ Movement «, Interactions, Performance, » laboratory (EA 4334), Nantes Université, Nantes, \\ France \\ ${ }^{4}$ Institut Universitaire de France (IUF), Paris, France \\ *Corresponding author: t.dick@uq.edu.au \\ Telephone: +61733652198 \\ Word Count: 3647 \\ Submission type: Original Article
}

All authors were directly involved in the preparation and conduction of this study. All authors contributed to the writing of the manuscript. The material in this manuscript will not be submitted for publication elsewhere.

Keywords: aging, B-mode ultrasound, elastography, shear modulus, stiffness, young's modulus 


\begin{abstract}
Ageing is associated with alterations in the structure and function of the contractile and elastic tissues that enable movement, posture, and balance. Alterations in structure and mechanical properties of the ankle plantarflexors and Achilles tendon are of particular interest due to their important 'catapultlike' function during efficient and healthy human locomotion. In this study, we examined age-related differences in the in vivo mechanical properties of both muscle and tendon in the human ankle plantarflexors in healthy younger $(21 \pm 3.25$ years $)$ and older $(69 \pm 2.86$ years $)$ adults. All participants were physically active, to represent healthy ageing. B-mode ultrasound coupled with force measurements was used to determine in vivo Achilles tendon stiffness and shear-wave elastography was used to measure shear modulus, an index of muscle stiffness, in the medial and lateral gastrocnemii. We found that older adults displayed 43\% lower $(\mathrm{p}=0.004)$ Achilles tendon stiffness, 59\% lower $(\mathrm{p}<0.001)$ Achilles tendon Young's modulus, and 34\% greater $(\mathrm{p}=0.002)$ Achilles tendon cross-sectional area compared to younger participants. We found no difference in the shear modulus of the medial or lateral gastrocnemii between the younger and older individuals. The reduction in Achilles tendon stiffness coupled with similar gastrocnemii muscle shear modulus likely influences the integrated neuromechanical function of the ankle plantarflexor muscle-tendon units during locomotor tasks. Further investigations into the relationship between altered mechanical properties and in vivo muscle-tendon dynamics will provide greater insights into the age-related declines in mobility and locomotor function.
\end{abstract}




\section{Introduction}

With age, muscle and tendon undergo alterations in architecture and composition that may impact on the mechanical properties and function of these tissues (Alnaqeeb et al., 1984; Narici et al., 2003; Tuite et al., 1997; Stenroth et al., 2012). Age-related differences in the mechanical properties of the extensor muscle-tendon units that cross the ankle (i.e. plantarflexors) are critically important for locomotion. Ultrasound imaging studies have revealed that the ankle powers walking via a 'catapult' mechanism within the Achilles tendon (AT) and its associated plantarflexor muscles (Ishikawa et al., 2005). In younger adults, this mechanism enables the ankle to provide $40-50 \%$ of the total positive lower limb mechanical power, propelling the body from one step to the next (Farris and Sawicki, 2012), however this reduces by up to 30\% in older adults (DeVita et al., 2000). In addition, walking in older adults is more energetically expensive (Peterson et al., 2010) and reduced ankle power has been identified as a key predictor of falls risk in ageing populations (Whipple et al., 1987). A comprehensive understanding of age-related differences in ankle plantarflexor muscle and tendon mechanical properties may assist in the development of strategies to prevent mobility deficits in older populations.

With advances in B-mode ultrasound imaging, the association between ageing and AT mechanical properties has been explored in recent decades. Although a high degree of inconsistency in findings exist (Delabastita et al., 2019), it is accepted that older individuals display reduced AT stiffness and AT Young's modulus compared to younger adults (Mademli and Arampatzis, 2008; Stenroth et al., 2012). However, there are no consistent differences reported in AT cross-sectional area (CSA), with conflicting reports on whether CSA increases (e.g., Stenroth et al., 2012) or decreases (Onambele et al., 2006). The discrepancy between studies is likely due to differences in experimental methodologies. Experimental studies have shown that plantarflexor muscle fascicle 
dynamics are altered in older adults during the gait cycle (Stenroth et al., 2017, Mian et al., 2007), and there is some evidence provided by simulation studies that age-related differences in AT stiffness lead to unfavourable muscle fascicle dynamics during walking (Franz and Thelen, 2016; Orselli et al., 2017). However, there are no other studies to our knowledge that have examined age-related differences in both muscle and tendon mechanical properties together, through a combination of shear wave elastography and B-mode ultrasound.

Age-related differences in the mechanical properties of human muscle in vivo remain poorly characterised. In vitro and in situ studies provide evidence that with age, human (Ochala et al., 2007) and animal (Danos et al., 2016; Holt et al., 2016) passive muscle fibre bundle stiffness increases. In addition, older adults display increased levels of intramuscular adipose tissue compared to younger adults (Addison et al., 2014), which has been suggested to lead to an increase in muscle stiffness and altered muscle mechanical output (Rahemi et al., 2015). Recently, shear wave elastography (SWE) has emerged as a non-invasive imaging technique that allows muscle shear modulus (an index of stiffness) to be estimated in vivo. Few studies have used SWE to quantify age-related differences in muscle shear modulus, and their results are inconclusive. Eby et al. (2015) measured shear modulus of the biceps brachii and found an increase in shear modulus with age, whereby others have measured shear wave velocity in the quadriceps, hamstrings, and the biceps brachii (Alfuraih et al., 2019) or shear modulus of the lateral gastrocnemius, soleus, and rectus femoris (Akagi et al., 2015) and shown lower values in the older age group. Thus, there are some conflicting results whereby in vitro, in situ, and some in vivo studies suggest an increase in muscle stiffness or shear modulus with age, and other in vivo findings suggest lower shear modulus values with age.

To date, we have a limited understanding of the age-related differences in mechanical properties of both muscle and tendon, as studies have commonly considered either muscle or tendon, 
but not both. However, the integrated mechanical function of both muscle and tendinous tissues is critical for efficient locomotor performance. Thus, the primary aim of this study was to determine age-related differences in the in vivo mechanical properties of the plantarflexor muscles and Achilles tendon. We hypothesised that AT stiffness and Young's modulus would be lower, and that shear modulus of the medial gastrocnemius (MG) and lateral gastrocnemius (LG) would be higher, in older compared to younger individuals.

\section{Methods}

Eighteen younger (18-35-yrs) and 22 older (65-75-yrs) adults participated in the study (Table 1). In an effort to isolate the effect of age from the effect of physical deconditioning, all participants were physically active, defined as $>600$ MET minutes per week, derived from the Active Australia Self-Report Physical Activity Measure. Participant exclusion criteria included recent (6 months) lower leg injury and any history of Achilles tendon pain. All participants provided informed consent and ethics approval was obtained from the University of Queensland (Approval \#2013001448).

\section{Tendon Mechanical Properties}

Participants performed ramped maximum voluntary isometric plantarflexion contractions (MVC) while seated in a custom-made frame with the ankle at 90 degrees and the knee at 130 degrees of extension (Dick et al., 2016). Straps were secured over the ankle and mid-thigh were used to minimise joint rotation during the contractions. A switch pressure-sensor under the heel was connected to an LED light and provided visual feedback to determine if the heel lifted during a contraction. The straps were tightened between each trial, and trials were repeated if the heel lifted. Participants were verbally encouraged during the MVCs and all measurements were made on the nondominant leg. 
Plantarflexion force was measured using a strain gauge (1020 Hz, FUTEK LSB350, USA), whilst B-mode ultrasound (7MHz, $60 \mathrm{~mm}$ field-of-view; MicroUs, Telemed, 45-50 Hz, Lithuania) simultaneously recorded the position of the MG muscle-tendon junction. Achilles tendon length change was calculated by manually digitising the position of the distal MG muscle-tendon junction (ImageJ, NIH, USA) (Fig. 1B). Tendon stiffness was calculated as the slope of a linear fit of the forceelongation curve between 10\% and $80 \%$ of MVC force (Dick et al., 2016) and Young's modulus as the slope of a linear fit of the stress-strain curve between $10 \%$ and $80 \%$ of MVC strain (Fig. 1A).

Stress $(\sigma)$ was calculated as $\sigma=\frac{F}{C S A}$ (F; plantarflexion force, CSA; Achilles tendon cross-sectional area), and strain ( $\varepsilon$ ) as $\varepsilon=\frac{l-l_{0}}{l_{0}}\left(1\right.$; tendon length, $1_{0}$; resting tendon length). Resting tendon length was determined using the ultrasound-tape method (Barber et al., 2011) with the ankle positioned at 90 degrees and the knee at 130 degrees. Briefly, this method involves the attachment of a tape measure to the ultrasound transducer placed over the MG MTJ at one end, and positioned over the Achilles tendon insertion on the calcaneus at the other end. This method has shown high reliability with 3D freehand ultrasound methods (Barber et al., 2011).

CSA of the AT was measured using B-mode ultrasound (Aixplorer, 4-15MHz, Vermon, Tours, France). Ultrasound images of the free tendon were taken in the transverse plane, at the midpoint between the AT insertion on the calcaneus and the soleus muscle-tendon junction. We used the polygon tool in Aixplorer to trace the border of the tendon inside the paratendon. CSA was calculated as the average of 2-4 measurements for each individual.

\section{Muscle Mechanical Properties}

Participants were prone with their non-dominant leg extended and ankle resting over the edge of the plinth. Their ankle angle in this position (defined herein as neutral) was measured using a 
handheld goniometer. SWE image sequences (Aixplorer, 4-15MHz, Vermon, Tours, France) of the MG and LG muscle bellies were acquired in triplicates at two different passive ankle positions: the neutral position and with the ankle held (using a fixed strap) in 15 degrees more dorsiflexion (i.e neutral +15 degrees (Slane et al., 2015)). During SWE recordings (5s), surface electromyography (EMG) of the MG, LG, soleus, and tibialis anterior was recorded $(2040 \mathrm{~Hz}$, Trigno Delsys Inc., Natick, MA, USA). Data were inspected visually during testing, and during the post processing period to ensure data is only reported from trials where the muscles were not active. SWE recordings were taken in the dosiflexed position $>60$ seconds after the ankle was secured in this position to minimize the effect of stress-relaxation on shear modulus values.

The technique used to measure the shear modulus has been described in detail (Gennisson et al., 2013). Briefly, SWE relies on the measurement of shear wave velocity that results from mechanical perturbations to the tissue. Shear modulus $(\mu)$ is related to the shear wave velocity (Vs): $\mu=\rho \mathrm{Vs}^{2}$, where $\rho$ is the density of the tissue $\left(1,000 \mathrm{~kg} / \mathrm{m}^{3}\right.$ for muscle). Data were processed using custom-written MATLAB scripts (MathWorks, Natick, MA, USA). Ultrasound videos exported from Aixplorer's software were sequenced into .jpeg image sequences. Each pixel of the colour map was converted into a shear modulus value established from an image-processing algorithm (Hug et al., 2013). Shear modulus values were averaged for five seconds over the largest region of interest that avoided aponeurosis and artifacts (Fig. 1C). We report shear modulus at neutral and neutral +15 degrees dorsiflexion as well as the change in muscle shear modulus associated with this 15 degree change in ankle angle.

Seven participants ( $\mathrm{n}=3$ and $\mathrm{n}=4$ for younger and older group, respectively) were excluded from tendon and force analysis due to poor-quality B-mode ultrasound or inability to perform MVCs, rendering MTJ tracking not possible, and ten $(\mathrm{n}=6$ and $\mathrm{n}=4$ for the younger and older group, 
respectively) were excluded from muscle analysis due to the presence of muscle activity during SWE measurements or void areas within the tissue region of interest.

\section{Statistical Analysis}

Two-tailed Welch's t-tests were performed to determine the effect of age on AT stiffness, AT Young's modulus, and participant characteristics. A Mann-Whitney test was performed if the data violated normality assumptions. A mixed-model ANOVA was performed to determine the influence of age (a between-subject factor), ankle position, and muscle (within-subject factors) on muscle shear modulus. Significance was set as $p<0.05$.

\section{Results}

There were no significant differences in body mass, BMI, maximum plantarflexion force, or METmin of weekly physical activity between the younger and older groups (all p $>0.089$, Table 1). Neutral ankle angle was approximately 4 degrees more plantarflexed in the older group $(\mathrm{p}=0.041$, Table 1).

Achilles tendon stiffness was $43 \%$ lower (younger $194.8 \pm 91.3 \mathrm{~N} / \mathrm{mm}$; older: $110.8 \pm 39.4$ N/mm, p=0.004, Fig 2A) and Young's modulus 59\% lower (younger: $0.76 \pm 0.40 \mathrm{GPa}$; older: $0.31 \pm$ $0.14 \mathrm{GPa}, \mathrm{p}<0.001$, Fig. $2 \mathrm{~B})$ in the older compared to the younger group. Achilles tendon crosssectional area was 34\% greater in older adults (younger: $50.9 \pm 10 . \mathrm{mm}^{2}$; older: $68.4 \pm 16.6 \mathrm{~mm}^{2}$, $\mathrm{p}=0.002$, Fig. 2C).

There was no significant difference in muscle shear modulus between the younger and older groups ( $\mathrm{p}=0.188$ ) (neutral position: $\mathrm{MG}$ younger: $3.78 \pm 0.91 \mathrm{kPa}, \mathrm{MG}$ older: $4.00 \pm 1.12 \mathrm{kPa}$; LG younger $2.82 \pm 1.14 \mathrm{kPa}$, LG older: $3.42 \pm 0.93 \mathrm{kPa}$; dorsiflexed position: MG younger: $7.82 \pm 1.3$ kPa, MG older: $9.08 \pm 2.99 \mathrm{kPa}$, LG younger: $6.39 \pm 1.10 \mathrm{kPa}, \mathrm{LG}$ older: $7.05 \pm 2.02 \mathrm{kPa}$ (Fig. 4). 
There was a significant main effect of ankle angle $(p<0.001)$ and muscle $(p<0.001)$, and an angle*muscle interaction $(\mathrm{p}<0.001)$ on muscle shear modulus. MG and LG shear modulus both increased in the dorsiflexed position compared to neutral (both $\mathrm{p}<0.001$ ). However, this change was greater in the MG (neutral: $3.92 \pm 1.04 \mathrm{kPa}$, dorsiflexed: $8.63 \pm 2.56 \mathrm{kPa}$ increase) compared to the LG (neutral: $3.19 \pm 1.04 \mathrm{kPa}$, dorsiflexed: $6.81 \pm 1.75 \mathrm{kPa}$ ) (Fig. 4). There was no significant difference, in the change in muscle shear modulus associated with a 15 degree change in ankle angle, between the older and younger groups $(\mathrm{p}=0.282)$.

\section{Discussion}

We aimed to determine if the mechanical properties of the human Achilles tendon and gastrocnemii muscles differ in older versus younger adults. Our groups were matched for physical activity level, and performed similarly in the maximum plantarflexion task. Despite similarities in physical performance, the older group had lower AT stiffness and Young's modulus. There were no differences in the shear modulus (as an index of muscle stiffness) of either gastrocnemii muscle.

The age-related changes in tendon properties are consistent with the findings of others (Csapo et al., 2014; Karamanidis et al., 2008; Onambele et al., 2006; Stenroth et al., 2012). Stenroth and collegues (2012) report 17\% lower AT stiffness and 32\% lower AT Young's modulus in older participants compared to young, which is less than the $59 \%$ and $43 \%$ difference for stiffness and Young's modulus, that we observed in this population. Intruigingly, the younger group displayed greater inter-individual variability in tendon stiffness and Young's modulus compared to the older group. This may reflect greater variation in either the type of physical activity or the intensity of the activity in the younger compared to the older group. CSA of the AT was larger in older individuals, which is similar to Stenroth et al. (2012), but in contrast with others (Onambele et al., 2006). It has 
been suggested that an increased tendon CSA in older adults could be the result of compositional changes, such as the accumulation of extracellular lipid deposits (Finlayson and Woods, 1975), while some suggest that an increase in CSA is a compensatory mechanism to counteract reduced tendon material properties with age (Stenroth et al., 2017). Based on the current data, there was a net reduction in stiffness, suggesting that increases in CSA did not fully offset the reduction in Young's modulus (Stenroth et al., 2012). In fact, Young's modulus varies considerably more than tendon stiffness and CSA, consistent with other studies (Stenroth et al., 2012; 2016), suggesting that ageing may have a greater influence on the instrinsic material properties of tendon, rather than its structural properties.

With age, mammalian tendons undergo a variety of alterations in composition and structure that influence mechanical function. Due to the invasive nature of experimental protocols, much of our knowledge regarding the mechanisms that underpin the effects of ageing on tendon properties are from non-human animal studies. However, the effects of ageing on tendon properties varies across these animal models, with older tendons displaying decreased elastin expression (rat) (Kostrominova and Brooks., 2013), decreased large-diameter collagen fibrils (rabbit) (Dressler et al., 2002; Nakagawa et al., 1994), or no difference in collagen fibril morphology (mice) (Wood et al., 2011). Similarly, studies that have compared tendon mechanical properties between young and older groups within the same animal model are inconclusive: similar to our results, the stiffness of rat AT has been shown to decrease (LaCroix et al., 2013; Vogel, 1978) but it has also been shown to increase with age (Danos et al., 2016; Nielsen et al., 1998; Wood et al., 2011). The high level of discrepancy between studies on the association between ageing and tendon properties makes direct comparisons with our results challenging. Differences may be related to the animal model, location/function of the tendon, or experimental methodology. 
The collagen concentration in skeletal muscle of non-human animals has been shown to increase with age (Alnaqeeb et al., 1984; Zimmerman et al., 1993), which should lead to an increase in stiffness. However, studies of collagen concentrations in human skeletal muscle suggest collagen levels are similar between younger and older participants (Babraj et al., 2005; Haus et al., 2007). The age-related increase in intramuscular adipose tissue (Addison et al., 2014) has been suggested to lead to an increase in muscle stiffness and altered muscle mechanical output (Rahemi et al., 2015). However, we did not find a significant difference in shear modulus in the MG or LG between older and younger individuals. This is in disagreement with other SWE studies, which find either higher shear modulus (Eby et al., 2015) or lower shear modulus or shear wave velocity (Akagi et al., 2015; Alfuraih et al., 2019) in older adults. Two studies that used alternative methods, vibro-ultrasound (Wang et al., 2014) or magnetic resonance elastography (Domire et al., 2009), to measure shear modulus in vivo found similar results to ours, with no difference in vastus intermedius or tibialis anterior shear modulus between younger and older participants.

For our study, in an effort to uncouple the effects of ageing from the combined effects of ageing and disuse, we recruited individuals who performed regular physical activity. Indeed there was no significant difference in MET minutes between older and younger groups, and our two groups performed similarly in their maximum voluntary efforts (Table 1). Previous studies that have examined the influence of age on muscle stiffness in humans either did not specify physical activity levels as an eligibility criteria (Eby et al. 2015; Alfuraih et al. 2019) or included a combination of sedentary and active participants (Akagi et al. 2015). It has been established that exercise can counteract some of the age-related declines in muscle-tendon unit function (Tieland et al., 2018). Thus, it is possible that the discrepancy between our results and previous studies that have examined the association between ageing and muscle properties is related to the difference in physical activity 
profiles between participants. This is further supported by Alfuraih et al. (2019) who found muscle strength was lower in older adults, and that irrespective of age, there was a positive correlation between muscle strength and shear wave velocity. Both Eby et al. (2015) and Akagi et al. (2015) do not report muscle strength.

Here, we found an age-related decrease in tendon stiffness, with no difference in muscle shear modulus. One possible contributor to these results is the type of physical activity. Although the weekly MET minutes did not differ between groups, on average, the older adults performed $\sim 20 \%$ less vigorous exercise compared to the younger adults. This difference in exercise intensity between the two cohorts may help to explain the mismatch in tendon and muscle properties. Tendons require greater loads to trigger adaptation than muscles. For example, studies have found that high strains $(>90 \%$ MVC, $4.5-5.0 \%$ tendon strain) are required for tendon adaptation, but muscles have been shown to adapt even with low intensity exercise ( $>30 \%$ MVC) (Arampatzis et al., 2010; Burd et al., 2012). Studies that have examined the effects of both low and high intensity exercise on the mechanical properties of tendon have found that although both protocols lead to increases in maximum plantarflexion force, it is only the high intensity exercise that leads to increases in tendon stiffness (Arampatzis et al., 2007; Arampatzis et al., 2010). Similar results have also been demonstrated in older adults (Grosset et al., 2014), athough some inconsistent findings exist. Stenroth et al. (2016) found no differences in tendon stiffness between older endurance athletes, sprint trained athletes, and older control participants. However, all older groups displayed larger tendon CSA and lower Young's modulus compared to younger adults (Stenroth et al., 2016). This is of importance because age-related differences in tendon stiffness and plantarflexion strength have been shown to lead to a reduction in the ability to restore balance after a forward fall, and that physical activity may be able to partly compensate for this in an older population (Karamanidis and Arampatzis, 2006). 
In this study we measured tendon properties under active tensile loading - a physiologically relevant loading task to locomotion. However, shear modulus, an index of muscle stiffness, was determined under a resting state. This approach is consistent with studies that aim to assess the instrinsic mechanical properties of muscle (e.g.; Maïsetti et al., 2012; Akagi et al., 2015; Eby et al., 2015; Le Sant et al., 2019), and eliminates the well known influence of muscle activation on shear modulus that occurs during active contractions (Nordez and Hug, 2010). We acknowledge that our study is not without limitations. First, we used B-mode ultrasound to determine AT CSA, which has been suggested to underestimate CSA values in comparison to the gold standard magnetic resonance imaging, and may contribute to the lack of consensus surrounding age-related differences in AT CSA (Bohm et al., 2016; McCrum et al., 2018). As a result of this, we may overestimate the age-related differences in tendon stiffness and Young's modulus leading to values more consistent with those reported in previous studies (Stenroth et al., 2012; Karamanidis et al., 2008). Second, we used an LED light to determine if the heel lifted during the MVC trials, however previous studies have shown that straps around the ankle joint do not completely prevent the rotations that occur when performing MVCs. As such, the forces measured by the strain gauge and the moments around the ankle joint in the same plane can differ, resulting in a possible overestimation of tendon elongation (Arampatzis et al., 2005). It should be acknowledged that muscle shear modulus was not measured in a standardised angle for each individual in our study, and the neutral angle was more dorsiflexed for the older than younger group.

In this study we used shear-wave elastography and B-mode ultrasound to determine the influence of ageing on muscle and tendon properties. We provide evidence for an age-related decrease in tendon stiffness and Young's modulus, but no difference in muscle shear modulus between younger and older individuals. The age-related reduction in tendon stiffness coupled with similar muscle shear 
modulus likely influences the neuromechanical behaviour of the plantarflexor muscle-tendon units during locomotion. For example, age-related reductions in AT stiffness would lead to more tendon stretch for a given load under similar kinematic trajectories (and thus muscle-tendon unit lengths). This would result in a concomitant reduction in the operating lengths of plantarflexor muscle fascicles, causing them to operate at shorter lengths (further from the optimal 'plateau' of the forcelength relationship), thereby requiring greater muscle activations to power locomotion. This mechanism is supported with model predictions (Franz and Thelen, 2016; Orselli et al., 2017), but remains to be experimentally validated. Future studies that combine in vivo measurements of mechanical properties, together with muscle fascicle and tendon dynamics during movement, will provide greater insight into the age-related declines in locomotor function. This knowledge has the potential to guide the design of targeted interventions to restore tendon stiffness and preserve independent mobility in later years.

\section{Conflict of interest statement.}

There are no conflicts of interest.

\section{Acknowledgements}

We acknowledge the support from a University of Queensland Early Career Fellowship awarded to TJM Dick. We also thank Anna Gore for assistance during data collection.

\section{References}

Addison, O., Drummond, M., LaStayo, P., Dibble, L., Wende, A., McClain, D., Marcus, R., 2014. Intramuscular fat and inflammation differ in older adults: the impact of frailty and inactivity. The Journal of Nutrition, Health and Aging 18, 532-538.

Alfuraih, A.M., Tan, A.L., O'Connor, P., Emery, P., Wakefield, R.J., 2019. The effect of ageing on shear wave elastography muscle stiffness in adults. Aging Clinical and Experimental Research 31, 19. 
Akagi, R., Yamashita, Y., Ueyasu, Y., 2015. Age-related differences in muscle shear moduli in the lower extremity. Ultrasound in Medicine and Biology 41, 2906-2912.

Alnaqeeb, M., Al Zaid, N., Goldspink, G., 1984. Connective tissue changes and physical properties of developing and ageing skeletal muscle. Journal of Anatomy 139.

Arampatzis, A., Karamanidis, K., Albracht, K., 2007. Adaptational responses of the human Achilles tendon by modulation of the applied cyclic strain magnitude. Journal of Experimental Biology 210, 2743-2753.

Arampatzis, A., Morey-Klasping, G., Karamanidis, K., DeMonte, G., Stafilidis, S., Brüggemann, G.P., 2005. Differences between measured and resultant joint moments during isometric contractions at the ankle joint. Journal of Biomechanics 38, 885-892.

Arampatzis, A., Peper, A., Bierbaum, S., Albracht, K., 2010. Plasticity of human Achilles tendon mechanical and morphological properties in response to cyclic strain. Journal of Biomechanics 43, 3073-3079.

Babraj, J.A., Cuthbertson, D.J., Smith, K., Langberg, H., Miller, B., Krogsgaard, M.R., Kjaer, M., Rennie, M.J., 2005. Collagen synthesis in human musculoskeletal tissues and skin. American Journal of Physiology-Endocrinology and Metabolism 289, 864-869.

Barber, L., Barrett, R., \& Lichtwark, G. (2011). Validity and reliability of a simple ultrasound approach to measure medial gastrocnemius muscle length. Journal of anatomy, 218(6), 637-642.

Bohm, S., Mersmann, F., Schroll, A., Mäkitalo, N., Arampatzis, A., 2016. Insufficient accuracy of the ultrasound-based determination of Achilles tendon cross-sectional area. Journal of biomechanics 49, 2932-2937.

Burd, N.A., Mitchell, C.J., Churchward-Venne, T.A., Phillips, S.M., 2012. Bigger weights may not beget bigger muscles: evidence from acute muscle protein synthetic responses after resistance exercise. Applied physiology, nutrition, and metabolism 37, 551-554.

Csapo, R., Malis, V., Hodgson, J., Sinha, S., 2014. Age-related greater Achilles tendon compliance is not associated with larger plantar flexor muscle fascicle strains in senior women. Journal of Applied Physiology 116, 961-969.

Danos, N., Holt, N.C., Sawicki, G.S., Azizi, E., 2016. Modeling age-related changes in muscle-tendon dynamics during cyclical contractions in the rat gastrocnemius. Journal of Applied Physiology 121, 1004-1012.

Delabastita, T., Bogaerts, S., Vanwanseele, B., 2019. Age-Related Changes in Achilles Tendon Stiffness and Impact on Functional Activities: A Systematic Review and Meta-Analysis. Journal of Aging and Physical Activity 27, 116-127.

DeVita, P., Hortobagyi, T., 2000. Age causes a redistribution of joint torques and powers during gait. Journal of Applied Physiology 88, 1804-1811.

Dick, T.J.M., Arnold, A.S., Wakeling, J.M., 2016. Quantifying Achilles tendon force in vivo from ultrasound images. Journal of Biomechanics 49, 3200-3207. 
Domire, Z.J., McCullough, M.B., Chen, Q., An, K.-N., 2009. Feasibility of using magnetic resonance elastography to study the effect of aging on shear modulus of skeletal muscle. Journal of applied biomechanics 25, 93-97.

Dressler, M., Butler, D., Wenstrup, R., Awad, H., Smith, F., Boivin, G., 2002. A potential mechanism for age-related declines in patellar tendon biomechanics. Journal of Orthopaedic Research 20, 13151322.

Eby, S.F., Cloud, B.A., Brandenburg, J.E., Giambini, H., Song, P., Chen, S., LeBrasseur, N.K., An, K., 2015. Shear wave elastography of passive skeletal muscle stiffness: influences of sex and age throughout adulthood. Clinical Biomechanics 30, 22-27.

Farris, D. J., \& Sawicki, G. S., 2012. The mechanics and energetics of human walking and running: a joint level perspective. Journal of The Royal Society Interface 9(66), 110-118.

Finlayson, R., \& Woods, S.J., 1975. Lipid in the Achilles' tendon: A comparative study. Atherosclerosis 21(3), 371-389.

Franz, J.R., Thelen, D.G., 2016. Imaging and simulation of Achilles tendon dynamics: Implications for walking performance in the elderly. Journal of Biomechanics 49, 1403-1410.

Gennisson, J., Deffieux, T., Fink, M., Tanter, M., 2013. Ultrasound elastography: principles and techniques. Diagnostic and Interventional Imaging 94, 487-495.

Grosset, J., Breen, L., Stewart, C.E., Burgess, K.E., Onambele, G.L., 2014. Influence of exercise intensity on training-induced tendon mechanical properties changes in older individuals. Age 36.

Haus, J.M., Carrithers, J.A., Trappe, S.W., Trappe, T.A., 2007. Collagen, cross-linking, and advanced glycation end products in aging human skeletal muscle. Journal of Applied Physiology 103, 20682076.

Holt, N. C., Danos, N., Roberts, T. J., Azizi, E., 2016. Stuck in gear: age-related loss of variable gearing in skeletal muscle. Journal of Experimental Biology 219(7), 998-1003.

Hug, F., Lacourpaille, L., Maïsetti, O., \& Nordez, A. (2013). Slack length of gastrocnemius medialis and Achilles tendon occurs at different ankle angles. Journal of biomechanics, 46(14), 2534-2538.

Ishikawa, M., Komi, P.V., Grey, M.J., Lepola, V., Bruggemann, G., 2005. Muscle-tendon interaction and elastic energy usage in human walking. Journal of Applied Physiology 99, 603-608.

Karamanidis, K., Arampatzis, A., 2006. Age-related degeneration in leg-extensor muscle-tendon units decreases recovery performance after a forward fall: compensation with running experience. European Journal of Applied Physiology 99, 73-85.

Karamanidis, K., Arampatzis, A., Mademli, L., 2008. Age-related deficit in dynamic stability control after forward falls is affected by muscle strength and tendon stiffness. Journal of Electromyography and Kinesiology 18, 980-989.

Kostrominova, T.Y., Brooks, S.V., 2013. Age-related changes in structure and extracellular matrix protein expression levels in rat tendons. Age 35, 2203-2214. 
LaCroix, A.S., Duenwald-Kuehl, S.E., Brickson, S., Akins, T.L., Diffee, G., Aiken, J., Vanderby, R., Lakes, R.S., 2013. Effect of age and exercise on the viscoelastic properties of rat tail tendon. Annals of biomedical engineering 41, 1120-1128.

Le Sant, G., Nordez, A., Hug, F., Andrade, R., Lecharte, T., McNair, P. J., \& Gross, R. (2019). Effects of stroke injury on the shear modulus of the lower leg muscle during passive dorsiflexion. Journal of Applied Physiology, 126(1), 11-22.

Mademli, L., Arampatzis, A., 2008. Mechanical and morphological properties of the triceps surae muscle-tendon unit in old and young adults and their interaction with a submaximal fatiguing contraction. Journal of Electromyography and Kinesiology 18, 89-98.

Maïsetti, O., Hug, F., Bouillard, K., \& Nordez, A. (2012). Characterization of passive elastic properties of the human medial gastrocnemius muscle belly using supersonic shear imaging. Journal of biomechanics, 45(6), 978-984.

McCrum, C., Leow, P., Epro, G., König, M., Meijer, K., Karamanidis, K., 2018. Alterations in leg extensor muscle-tenon unit biomechanical properties with ageing and mechanical loading. Frontiers in Physiology 9.

Mian, O.S., Thom, J.M., Ardigò, L.P., Minetti, A.E., Narici, M.V., 2007. Gastrocnemius muscletendon behaviour during walking in young and older adults. Acta Physiologica 189, 57-65.

Morrison, S. M., Dick, T. J., \& Wakeling, J. M. (2015). Structural and mechanical properties of the human Achilles tendon: Sex and strength effects. Journal of biomechanics, 48(12), 3530-3533.

Nakagawa, Y., Majima, T., Nagashima, K., 1994. Effect of ageing on ultrastructure of slow and fast skeletal muscle tendon in rabbit Achilles tendons. Acta physiologica scandinavica 152, 307-313.

Narici, M.V., Maganaris, C.N., Reeves, N.D., Capodaglio, P., 2003. Effect of aging on human muscle architecture. Journal of Applied Physiology 95, 2229-2243.

Nielsen, H.M., Skalicky, M., Viidik, A., 1998. Influence of physical exercise on aging rats. III. Lifelong exercise modifies the aging changes of the mechanical properties of limb muscle tendons. Mechanisms of ageing and development 100, 243-260.

Nordez, A., \& Hug, F. (2010). Muscle shear elastic modulus measured using supersonic shear imaging is highly related to muscle activity level. Journal of applied physiology, 108(5), 1389-1394.

Ochala, J., Frontera, W.R., Dorer, D.J., Hoecke, J.V., Krivickas, L.S., 2007. Single skeletal muscle fiber elastic and contractile characteristics in young and older men. The Journals of Gerontology Series A: Biological Sciences and Medical Sciences 62, 375-381.

Onambele, G.L., Narici, M.V., Maganaris, C.N., 2006. Calf muscle-tendon properties and postural balance in old age. Journal of Applied Physiology 100, 2048-2056.

Orselli, M. I. V., Franz, J. R., \& Thelen, D. G. (2017). The effects of Achilles tendon compliance on triceps surae mechanics and energetics in walking. Journal of biomechanics, 60, 227-231.

Peterson, D.S., Martin, P.E., 2010. Effects of age and walking speed on coactivation and cost of walking in healthy adults. Gait and Posture 31, 355-359. 
Rahemi, H., Nigam, N., Wakeling, J.M., 2015. The effect of intramuscular fat on skeletal muscle mechanics: implications for the elderly and obese. Journal of The Royal Society Interface 12.

Slane, L. C., DeWall, R., Martin, J., Lee, K., \& Thelen, D. G. (2015). Middle-aged adults exhibit altered spatial variations in Achilles tendon wave speed. Physiological measurement, 36(7), 1485.

Stenroth, L., Cronin, N.J., Peltonen, J., Korhonen, M.T., Sipilä, S., Finni, T., 2016. Triceps surae muscle-tendon properties in older endurance- and sprint-trained athletes. Journal of Applied Physiology 120(1), 63-69.

Stenroth, L., Sipilä, S., Finni, T., Cronin, Neil., 2017. Slower walking speed in older men improved triceps surae force generation ability. Medicine and Science in Sports and Exercise 49, 158-166.

Stenroth, L., Peltonen, J., Cronin, N.J., Sipilä, S., Finni, T., 2012. Age-related differences in Achilles tendon properties and triceps surae muscle architecture in vivo. Journal of Applied Physiology 113, 1537-1544.

Svensson, R.B., Heinemeier, K.M., Couppé, C., Kjaer, M., Magnusson, S.P., 2016. Effect of aging and exercise on the tendon. Journal of applied physiology 121, 1353-1362.

Tieland, M., Trouwborst, I., Clark, B.C., 2018. Skeletal muscle performance and ageing. Journal of cachexia, sarcopenia and muscle 9, 3-19.

Tuite, D., Renström, P., O'Brien, M., 1997. The aging tendon. Scandinavian Journal of Medicine and Science in Sports 7, 72-77.

Vogel, H., 1978. Influence of maturation and age on mechanical and biochemical parameters of connective tissue of various organs in the rat. Connective tissue research 6, 161-166.

Wang, C.Z., Li, T.J., Zheng, Y.P., 2014. Shear modulus estimation on vastus intermedius of elderly and young females over the entire range of isometric contraction. PLoS One 9.

Whipple, R., Wolfson, L., Amerman, P., 1987. The relationship of knee and ankle weakness to falls in nursing home residents: an isokinetic study. Journal of the American Geriatrics Society 35, 13-20.

Wood, L.K., Arruda, E.M., Brooks, S.V., 2011. Regional stiffening with aging in tibialis anterior tendons of mice occurs independent of changes in collagen fibril morphology. Journal of applied physiology 111, 999-1006.

Zimmerman, S.D., McCormick, R.J., Vadlamudi, R.K., Thomas, D.P., 1993. Age and training alter collagen characteristics in fast-and slow-twitch rat limb muscle. Journal of Applied Physiology 75, 1670-1674. 


\section{Figures}
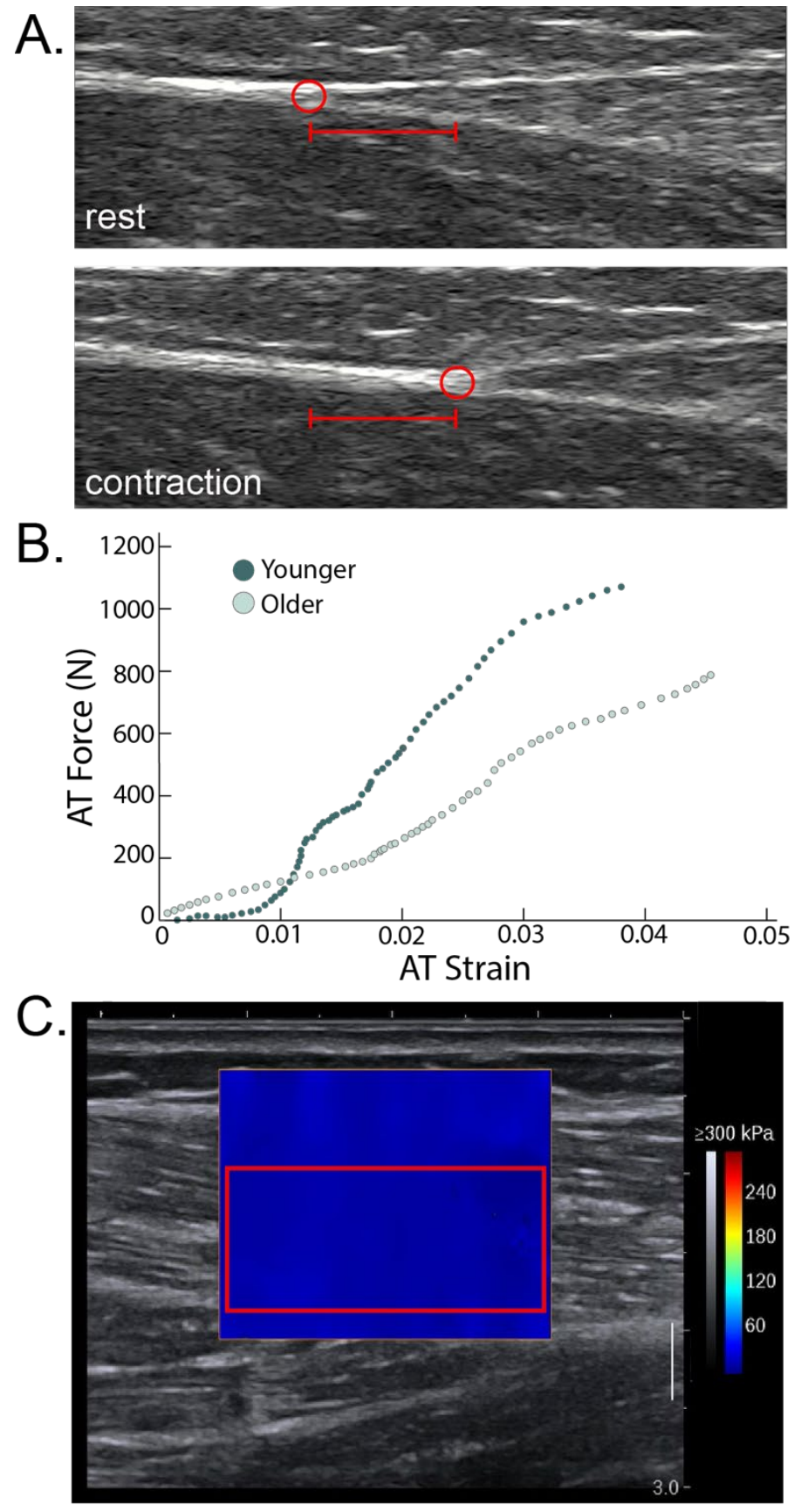

Fig. 1 Experimental methods. (A) B-mode ultrasound of gastrocnemius medialis (MG) muscletendon junction, at rest and during a contraction. Circles indicate position of MG muscle-tendon junction, hortizontal bars indicate total muscle-tendon junction displacement; (B) Achilles tendon force versus strain during ramped isometric plantarflexion contraction for one representative younger (darker) and older (lighter) participant. (C) Shear wave elastography of the MG. A shear modulus heat map is superimposed over a B-mode ultrasound image, the red rectangle indicates the region-ofinterest chosen to avoid aponeurosis and artifacts. Scale bar indicates shear modulus values for colors of the heat map. 
A. Achilles tendon stiffness

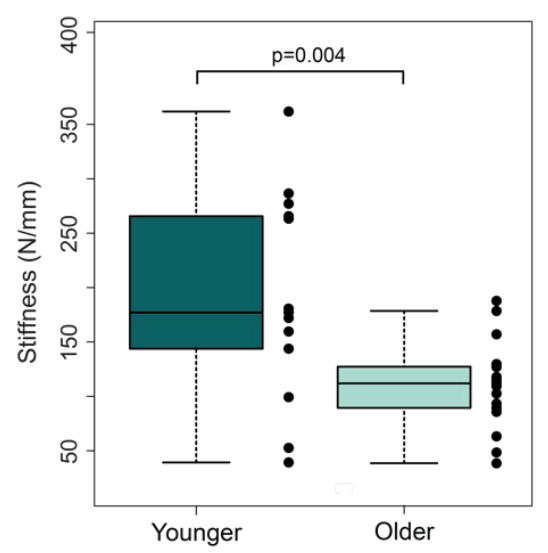

B. Achilles tendon Young's Modulus

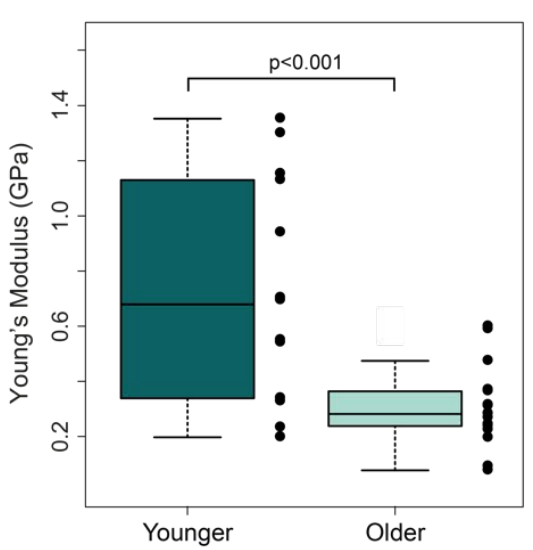

C. Achilles tendon cross-sectional area

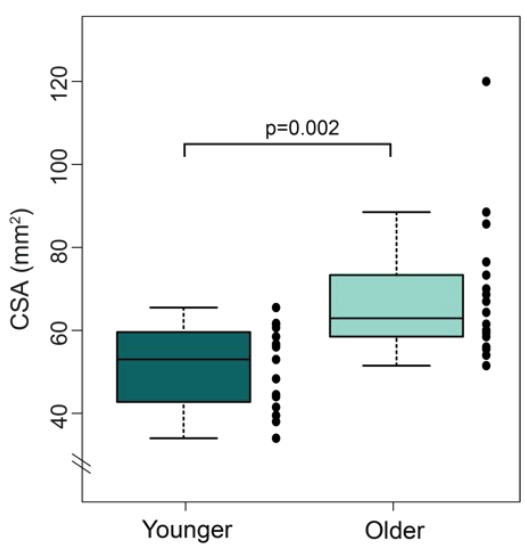

Fig. 2 (A) Achilles tendon stiffness and (B) Young's modulus were significantly lower in older adults compared to younger adults. (C) Achilles tendon cross-sectional area was significantly higher in older adults compared to younger adults.

A. Medial gastrocnemius

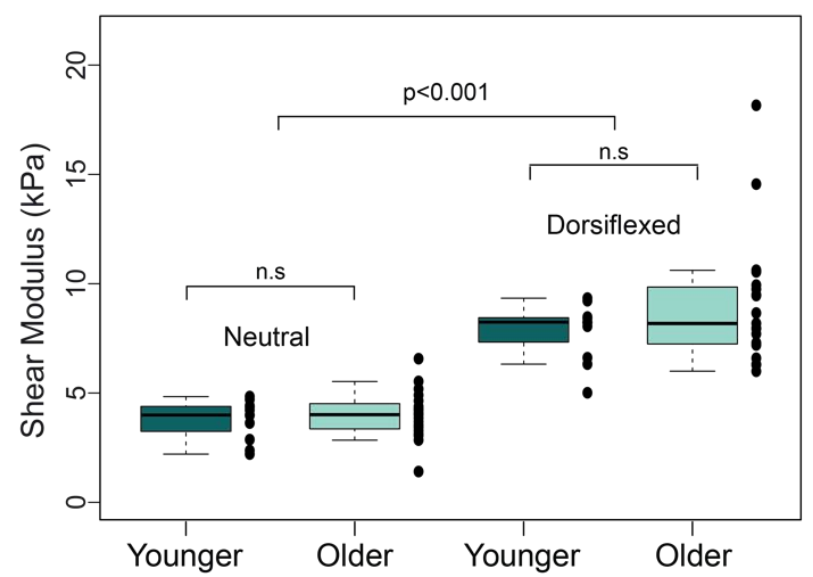

B. Lateral gastrocnemius

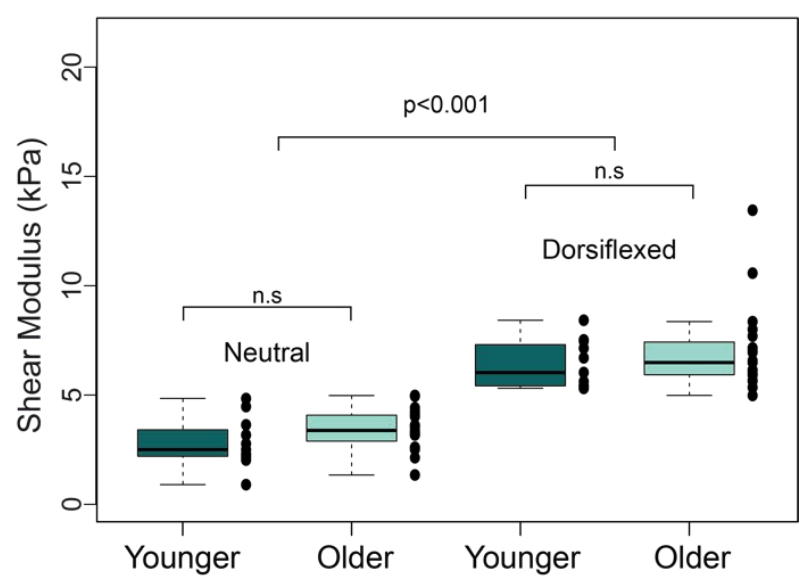

Fig. 3 Shear modulus, measured using shear wave elastography for the (A) medial and (B) lateral gastrocnemius, in younger and older adults. Muscle shear modulus was measured at two different ankle positions: neutral and $+15^{\circ}$ of dorsiflexion. The shear modulus was greater in dorsiflexion than neutral for both muscles. There was no difference in shear modulus between the younger and older groups. In addition to that noted on the figure, the change in shear modulus when the muscle was passively stretched from neutral to $+15^{\circ}$ of dorsiflexion was greater for the MG than the LG $(\mathrm{p}=0.028)$. 


\section{Tables}

Table 1 Participant Characteristics

\begin{tabular}{lcccccc}
\hline & $\begin{array}{c}\text { Age } \\
(\mathbf{y r s})\end{array}$ & $\begin{array}{c}\text { Body Mass } \\
\mathbf{( k g )}\end{array}$ & $\begin{array}{c}\text { BMI } \\
\left(\mathbf{k g} / \mathbf{m}^{2}\right)\end{array}$ & $\begin{array}{c}\text { Plantarflexion } \\
\text { MVC (N) }\end{array}$ & $\begin{array}{c}\text { Neutral } \\
\text { Ankle Angle } \\
\text { (deg.) }\end{array}$ & $\begin{array}{c}\text { Physical } \\
\text { Activity Level } \\
\text { (METmins/wk) }\end{array}$ \\
\hline Younger (n=18) & $21 \pm 3.25$ & $71 \pm 10.73$ & $23 \pm 3.54$ & $1135 \pm 314.63$ & $61 \pm 7.31$ & $3776 \pm 2442.57$ \\
\hline Older (n=22) & $69 \pm 2.86$ & $70 \pm 14.13$ & $24 \pm 3.36$ & $918 \pm 381.87$ & $65 \pm 3.35$ & $3260 \pm 1866.94$ \\
\hline $\mathbf{p}=$ & $<\mathbf{0 . 0 0 1}$ & 0.572 & 0.497 & 0.089 & $\mathbf{0 . 0 4 1}$ & 0.673
\end{tabular}

Values are expressed as mean \pm SD. BMI, body mass index; MVC, maximal voluntary contraction. 